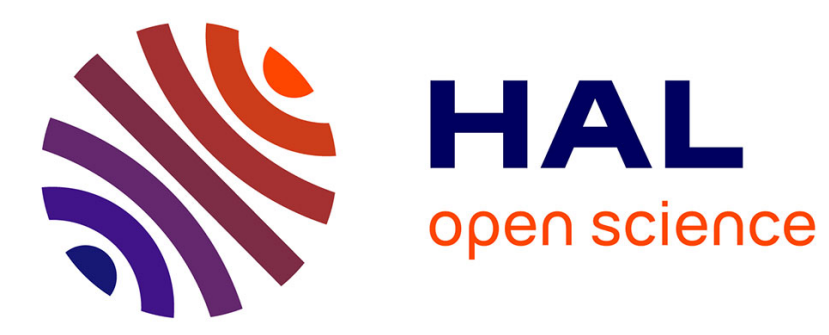

\title{
Localized waves in nonlinear oscillator chains
}

Gérard Iooss, Guillaume James

\section{To cite this version:}

Gérard Iooss, Guillaume James. Localized waves in nonlinear oscillator chains. Chaos: An Interdisciplinary Journal of Nonlinear Science, 2005, 15 (1), pp.015113. 10.1063/1.1836151 . hal-00012887

\section{HAL Id: hal-00012887 \\ https://hal.science/hal-00012887}

Submitted on 28 Oct 2005

HAL is a multi-disciplinary open access archive for the deposit and dissemination of scientific research documents, whether they are published or not. The documents may come from teaching and research institutions in France or abroad, or from public or private research centers.
L'archive ouverte pluridisciplinaire HAL, est destinée au dépôt et à la diffusion de documents scientifiques de niveau recherche, publiés ou non, émanant des établissements d'enseignement et de recherche français ou étrangers, des laboratoires publics ou privés. 


\title{
Localized waves in nonlinear oscillator chains
}

\author{
Gérard Iooss †, Guillaume Jamesł \\ $\dagger$ Institut Universitaire de France, INLN, UMR CNRS-UNSA 6618, \\ 1361 route des Lucioles, F-06560 Valbonne, France. \\ $\ddagger$ Laboratoire Mathématiques pour l'Industrie et la Physique (UMR 5640), \\ INSA de Toulouse, 135 avenue de Rangueil, 31077 Toulouse Cedex 4, France.
}

\begin{abstract}
This paper reviews and extends existence results for spatially localized waves in nonlinear chains of coupled oscillators. The models we consider are referred as Fermi-Pasta-Ulam (FPU) or KleinGordon (KG) lattices, depending whether nonlinearity takes the form of an anharmonic nearestneighbors interaction potential or an on-site potential. Localized solutions include solitary waves of permanent form $[20,24,27,29]$, and travelling breathers which appear time periodic in a system of reference moving at constant velocity. Approximate travelling breather solutions have been previously constructed in the form of modulated plane waves, whose envelopes satisfy the nonlinear Schrödinger equation [64], [51]. For KG chains and in the case of travelling waves (where the phase velocity of the plane wave equals the group velocity of the wave packet), the existence of nearby exact solutions has been proved by Iooss and Kirchgässner, who have obtained exact solitary wave solutions superposed on an exponentially small periodic tail. By a center manifold reduction they reduce the problem locally to a finite dimensional reversible system of ordinary differential equations, which admits homoclinic solutions to periodic orbits. It has been recently shown by James and Sire $[36,57]$ that the center manifold approach initiated by Iooss and Kirchgässner is still applicable when the breather period and the inverse group velocity are commensurate. The particular case when the breather period equals twice the inverse group velocity has been worked out explicitly for KG chains, and yields the same type of reduced system as for travelling waves if the on-site potential is symmetric. In that case, the existence of exact travelling breather solutions superposed on an exponentially small periodic tail has been proved. In this paper we apply the same method to the FPU system and treat the commensurate case in full generality (we give the main steps of the analysis and shall provide the details in a forthcoming paper [34]). We reduce the problem locally to a finite dimensional reversible system of ordinary differential equations, whose dimension can be arbitrarily large and is of the order of the number of resonant phonons. Its principal part is integrable, and admits solutions homoclinic to quasi-periodic orbits if a hardening condition on the potential is satisfied. These orbits correspond to approximate travelling breather solutions superposed to a quasi-periodic oscillatory tail. The problem of their existence for the full system is still open in the general case, and constitutes the final step for proving the existence of exact travelling breather solutions. In the particular case of an even potential and if the breather period equals twice the inverse group velocity, we prove indeed the existence of exact travelling breather solutions superposed to an exponentially small periodic tail.
\end{abstract}

Keywords: Fermi-Pasta-Ulam lattice, travelling breathers, nonlinear advance-delay differential equations, center manifold reduction.

\section{MODELS AND LITERATURE REVIEW}

We consider one-dimensional lattices described by the system

$\frac{d^{2} u_{n}}{d t^{2}}+W^{\prime}\left(u_{n}\right)=V^{\prime}\left(u_{n+1}-u_{n}\right)-V^{\prime}\left(u_{n}-u_{n-1}\right), n \in \mathbb{Z}$

where $u_{n}$ is the displacement of the $n$th particle from an equilibrium position. This system describes a chain of particles nonlinearly coupled to their first neighbors, in a local anharmonic potential. The interaction potential $V$ and on-site potential $W$ are assumed analytic in a neighborhood of $u=0$, with $V^{\prime}(0)=W^{\prime}(0)=0$, $V^{\prime \prime}(0), W^{\prime \prime}(0)>0$. System (1) is referred as Fermi-PastaUlam (FPU) lattice [13] for $W=0$ and Klein-Gordon (KG) lattice if $V$ is harmonic $\left(V(x)=\frac{\gamma}{2} x^{2}\right)$. These models have been used for the description of a broad range of physical phenomena, such as crystal dislocation [40], localized excitations in ionic crystals [55], thermal denat- uration of DNA [9].

In this paper, we consider solutions of (1) satisfying

$$
u_{n}(t)=u_{n-p}(t-p \tau),
$$

for a fixed integer $p \geq 1$ ( $p$ being the smallest possible) and $\tau \in \mathbb{R}$. The case when $p=1$ in (2) corresponds to travelling waves with velocity $1 / \tau$. Solutions satisfying (2) for $p \neq 1$ consist of pulsating travelling waves, which are exactly translated by $p$ sites after a fixed propagation time $p \tau$ and are allowed to oscillate as they propagate on the lattice. Solutions of type (2) having the additional property of spatial localization $\left(u_{n}(t) \rightarrow 0\right.$ as $\left.n \rightarrow \pm \infty\right)$ are known as exact travelling breathers (with velocity $1 / \tau)$ for $p \geq 2$ and solitary waves for $p=1$.

\section{A. Exact and approximate travelling breathers}

Approximate travelling breather solutions propagating on the lattice at a non constant velocity have drawn a 
lot of attention. They have been numerically observed in various one-dimensional nonlinear lattices such as FPU lattices [62], [8], [52], [15], KG chains [10],[6] and the discrete nonlinear Schrödinger (DNLS) equation [14]. Other references are available in the review paper [17]. One way of generating approximate travelling breathers consists of "kicking" static breathers consisting of spatially localized and time periodic oscillations (see the basic papers [63], [44], [17], [33], [5] for more details on these solutions). Static breathers are put into motion by perturbation in the direction of a pinning mode [6]. The possible existence of an energy barrier that the breather has to overcome in order to become mobile has drawn a lot of attention, see e.g. [10], [6], [15], [38] and the review paper [54]. Approximate travelling breathers can be formally obtained via effective Hamiltonians, which approximately describe the motion of the breather center on the lattice, at a nonconstant velocity [45], [38].

It is a delicate task to examine the existence of exact travelling breathers using numerical computations. Indeed, these solutions might not exist without being superposed on a small nonvanishing oscillatory tail which violates the property of spatial localization. Solitary waves [6] and travelling breathers [58] superposed on a small oscillatory tail have been numerically observed in KG lattices. Numerical results indicate similar phenomena for the propagation of kinks [11], [53], [4]. Fine analysis of numerical convergence problems also suggests that different nonlinear lattices do not support exact solitary waves or travelling breathers in certain parameter regimes [61], [3].

Different situations have been considered for the existence of exact travelling breathers in various simpler models. On the one hand, exact travelling breathers can be explicitly computed in the integrable Ablowitz-Ladik lattice [1], and other examples of nonlinear lattices supporting exact travelling breathers can be obtained using an inverse method [16]. On the other hand,.travelling breather solutions of the Ablowitz-Ladik lattice are not robust under various non-Hamiltonian reversible perturbations as shown in [7].

\section{B. The multi-scale expansion approach}

Formal multi-scale expansions have been used by several authors to obtain travelling breather solutions of (1). The case of KG lattices has been treated by Remoissenet [51]. A multi-scale expansion provides an evolution equation for the envelopes of well-prepared initial conditions corresponding to modulated plane waves

$$
u_{n}(t)=\epsilon A\left(\epsilon^{2} t, \epsilon(n-c t)\right) e^{i(q n-\omega t)}+c . c .+O\left(\epsilon^{2}\right) .
$$

Here $\omega=\omega(q)$ is given by the dispersion relation for the linear phonons and $c=\omega^{\prime}(q)$ is the group velocity of the wave packet. The amplitude $A(s, \xi)$ satisfies the nonlinear Schrödinger (NLS) equation

$$
i \partial_{s} A=-\frac{1}{2} w^{\prime \prime}(q) \partial_{\xi}^{2} A+h|A|^{2} A,
$$

$h$ depending on $q$ and $V, W$. In the focusing case $w^{\prime \prime}(q) h<0$, the NLS equation admits sech-shaped solutions corresponding (at least formally) to travelling breather solutions

$$
u_{n}(t)=\epsilon \alpha \frac{e^{i(q n-\omega t)}}{\cosh (\epsilon(n-c t))}+c . c .+O\left(\epsilon^{2}\right)
$$

(a $O\left(\epsilon^{2}\right)$ correction to $\omega$ has been left in higher order terms). One can write alternatively $u_{n}(t)=u(n-c t, t)+$ $O\left(\epsilon^{2}\right)$ where

$$
u(\xi, t)=\epsilon \alpha \frac{e^{i q \xi}}{\cosh (\epsilon \xi)} e^{i \omega_{b} t}+c . c .
$$

and $\omega_{b}=q c-\omega$. The function $u(\xi, t)$ is localized in $\xi$ and time-periodic with frequency $\omega_{b}$ (denoted as travelling breather frequency).

The multi-scale approach has been used by Tsurui [64] and Flytzanis et al [18] for the FPU lattice. For system (1) with $V^{\prime \prime}(0)>0$ the validity of the nonlinear Schrödinger equation on large but finite time intervals has been proved recently by Giannoulis and Mielke [25], [26].

\section{Generalized solitary waves in Klein-Gordon lattices}

It is a challenging problem to determine if the approximate solutions (5) could constitute the principal part of exact travelling breather solutions of the Klein-Gordon system. This would imply that linear dispersion is balanced by nonlinear terms at any order in the above mentioned multi-scale expansion.

This problem has been solved by Iooss and Kirchgässner for the KG system in the case of travelling waves [29], where the phase velocity of the plane wave equals the group velocity of the wave packet i.e. $c=\omega / q$. In that case approximate solutions (5) read $u_{n}(t)=u(n-c t)+O\left(\epsilon^{2}\right)$. Travelling wave solutions of (1) with $V(x)=\frac{\gamma}{2} x^{2}$ (and $p=1$ in (2)) have the form $u_{n}(t)=u_{0}(t-n \tau)$ and are determined by the scalar advance-delay differential equation

$$
\frac{d^{2} u_{0}}{d t^{2}}+W^{\prime}\left(u_{0}\right)=\gamma\left(u_{0}(t+\tau)-2 u_{0}+u_{0}(t-\tau)\right) .
$$

Iooss and Kirchgässner have studied small amplitude solutions of (7) in different parameter regimes and have obtained in particular "nanopterons" (or generalized solitary waves) consisting of a solitary wave superposed to an exponentially small oscillatory tail. The leading order part of these solutions (excluding their tail) coincides with approximate solutions obtained via the NLS equation. 
Their analysis is based on a reduction to a center manifold in the infinite dimensional case as described in references [39], [49], [65]. Equation (7) is rewritten as a reversible evolution problem in a suitable functional space, and considered for parameter values $(\tau, \gamma)$ near a critical curve (defined by $1 / \tau=c=\omega / q$ ) where the imaginary part of the spectrum of the linearized operator consists of a pair of double eigenvalues and a pair of simple ones. Close to this curve, the pair of double eigenvalues splits in two pairs of hyperbolic eigenvalues with opposite nonzero real parts, which opens the possibility of finding solutions homoclinic to 0 .

Near these parameter values, the center manifold theorem reduces the problem locally to a reversible 6dimensional system of differential equations. The reduced system is put in a normal form which is integrable up to higher order terms. In some regions of the parameter space, the truncated normal form admits reversible orbits homoclinic to 0 , which bifurcate from the trivial state and correspond to approximate solutions of (7).

However, as it is shown by Lombardi for different classes of reversible systems [42], these solutions should not generically persist when higher order terms are taken into account in the normal form. The existence of corresponding travelling waves decaying exactly to 0 should be a codimension-1 phenomenon, the codimension depending on the number of pairs of purely imaginary eigenvalues (i.e. the number of resonant phonons) in the parameter regime considered by the authors (there is one pair of purely imaginary eigenvalues, in addition to hyperbolic ones). However, to confirm the nonexistence of reversible homoclinic orbit to 0 (close to a small amplitude homoclinic orbit of the truncated normal form) for a given choice of $W, \gamma, \tau$, one has to check the nonvanishing of a certain Melnikov function being extremely difficult to compute in practice [42].

Due to this codimension- 1 character, in a given system (7) (with fixed coupling constant $\gamma$ and on-site potential $W$ ) exact travelling wave solutions decaying to 0 at infinity might exist in the small amplitude regime, but for isolated values of the wave velocity $1 / \tau$.

Instead of orbits homoclinic to 0 , the full normal form admits orbits homoclinic to small periodic ones [42] (originating from the pair of purely imaginary eigenvalues). These solutions correspond to exact solitary wave solutions of (1) superposed on a small periodic oscillatory tail, which can be made exponentially small with respect to the central oscillation size (the minimal tail size should be generically nonzero for a given value of $(\tau, \gamma))$.

\section{Travelling breathers in Klein-Gordon lattices}

The above results do not cover the case of travelling breathers in which the wavepacket has different phase and group velocities. However, it has been recently shown by James and Sire [57], [36] that the center manifold approach initiated by Iooss and Kirchgässner is still applicable to the KG model when the travelling breather period and the inverse group velocity are commensurate i.e. $\omega_{b} / c=2 \pi m / p(m \in \mathbb{Z})$. In that case the principal part of (5) satisfies (2) with $\tau=1 / c$. For fixed $p \geq 2$, problem (1)-(2) with $V(x)=\frac{\gamma}{2} x^{2}$ reduces to the $p$-dimensional system of advance-delay differential equations

$$
\begin{aligned}
\frac{d^{2} u_{1}}{d t^{2}}+W^{\prime}\left(u_{1}\right)= & \gamma\left(u_{2}-2 u_{1}+u_{p}(t+p \tau)\right), \\
\frac{d^{2} u_{n}}{d t^{2}}+W^{\prime}\left(u_{n}\right)= & \gamma\left(u_{n+1}-2 u_{n}+u_{n-1}\right), \\
& n=2, \ldots, p-1, \\
\frac{d^{2} u_{p}}{d t^{2}}+W^{\prime}\left(u_{p}\right)= & \gamma\left(u_{1}(t-p \tau)-2 u_{p}+u_{p-1}\right) .
\end{aligned}
$$

The case $p=2$ in (8)-(10) has been worked out explicitly and corresponds to the situation when the breather period equals twice the inverse group velocity.

The case when $W$ is even yields the same type of reduced system for travelling breathers as for travelling waves. In this case, there exist exact travelling breather solutions superposed on an exponentially small periodic tail and satisfying $u_{n}(t)=-u_{n-1}(t-\tau)$.

For asymmetric potentials, the simplest homoclinic bifurcation yields a higher-dimensional (8-dimensional) reduced system, with a supplementary pair of simple imaginary eigenvalues of the linearized operator (the imaginary part of the spectrum consists of a pair of double eigenvalues and two pairs of simple ones). The reduced normal form of the system is reversible and integrable up to higher order terms. In some regions of the parameter space, the truncated normal form admits reversible orbits homoclinic to 0 , which bifurcate from the trivial state and correspond to approximate solutions of (8)-(10). These approximate solutions coincide at leading order with spatially localized modulated plane waves obtained via the NLS equation.

However, by analogy with results of Lombardi [42] it has been conjectured in [36] that these solutions do not generically persist when higher order terms are taken into account in the normal form. Persistence might be true if parameters $(\tau, \gamma$, and coefficients in the expansion of $W$ ) are chosen on a discrete collection of codimension- 2 submanifolds in this infinite dimensional space. For general parameter values, instead of orbits homoclinic to 0 one can expect the existence of reversible orbits homoclinic to exponentially small 2-dimensional tori, originating from the two additional pairs of simple purely imaginary eigenvalues. These solutions should constitute the principal part of exact travelling breather solutions of (1) superposed on a small quasi-periodic oscillatory tail. However, in order to obtain exact solutions one has to prove the persistence of the corresponding homoclinic orbits as higher order terms are taken into account in the normal form. This step is non-trivial and would require to generalize results of Lombardi [42] available when one pair of simple imaginary eigenvalues is removed. Another promising approach is developed in the recent work 
of Iooss and Lombardi [30] on polynomial normal forms with exponentially small remainder for analytic vector fields.

The general case $p \geq 2$ in (8)-(10) is analyzed in a work in progress [59] (this case is technically more difficult but the approach used in [57], [36] works as well).

When the travelling breather period and the inverse group velocity are incommensurate, the principal part of (5) is not exactly translated on the lattice after time $p / c$ but is modified by a spatial shift. The existence of exact travelling breather solutions of this type is still an open problem.

\section{E. Solitary waves and travelling breathers in Fermi-Pasta-Ulam lattices}

The center manifold reduction method has been also used in [27] for studying travelling waves in FPU lattices. Near the largest critical value of velocity $1 / \tau=\sqrt{V^{\prime \prime}(0)}$ (denoted as sound velocity), all small amplitude travelling waves are given by finite-dimensional reversible differential equations. In particular, solitary wave solutions have been obtained above (and near) the sound velocity (in addition, heteroclinic solutions connecting a stretched pattern with a compressed one have been obtained). The global existence of solitary waves in FPU models has been proved with other types of methods $[19,20,24,60]$. In the small amplitude limit these solutions can be described by the Korteweg-de Vries (KdV) equation [37, 56], or the modified $\mathrm{KdV}$ equation in degenerate cases.

In the FPU system there are no exponentially small phenomena preventing the existence of $\mathrm{KdV}$ solitary waves decaying exactly to 0 , unlike for the KG lattice. This difference is due to the fact that localized solutions in the KG model (rather described by the NLS equation) mix a slow envelope decay with additional fast oscillations of a plane wave (see [42, 43] for more details on this type of nonpersistence phenomena).

The stability (in appropriate exponentially weighted spaces) of solitary wave solutions on fixed low energy surfaces has been proved in recent works [21-23]. A localized perturbation of a supersonic solitary wave produces (asymptotically in time) a perturbation of the speed and phase of the wave, and a radiating part that travels slower than the wave and decays locally near it.

A discrete version of the center manifold method has been developped in $[32,33]$ and applied to the existence of small amplitude static breathers in FPU chains. The FPU system is rewritten as a (reversible) mapping in a loop space, the index $n$ playing the role of a discrete time. When the frequency of solutions is near the top of the phonon band, the system can be locally reduced to a (reversible) 2-dimensional mapping. Static breathers (corresponding to orbits of the reduced map homoclinic to 0) have been proved to exist when a certain hardening condition on the interaction potential is satisfied. The case of diatomic FPU chains has been examined [35] using the same method, and the existence of small amplitude breathers has been proved for arbitrary mass ratio and hard or soft potentials in various frequency regimes (extending previous results valid for large mass ratio [41]).

In this paper we study the existence of travelling breathers in the FPU system and treat the commensurate case (2) in full generality. Near critical parameter values $\tau=\tau_{k}$ (with $\tau_{k}>\left(V^{\prime \prime}(0)\right)^{-1 / 2}$ ), the center manifold theorem reduces the problem locally to a finite dimensional reversible system of ordinary differential equations, whose dimension can be arbitrarily large (the dimension is of the order of the number of resonant phonons). Its principal part is integrable, and admits solutions homoclinic to quasi-periodic orbits if a hardening condition on the interaction potential $V$ is satisfied. These orbits correspond to approximate travelling breather solutions (close to the NLS limit (5)) superposed on a quasi-periodic oscillatory tail. The problem of their persistence for the full reduced system is still open in the general case, and constitutes the final step for proving the existence of exact travelling breather solutions in FPU chains. Note that in the particular case of an even potential, and if the breather period equals twice the inverse group velocity $\left(p=2, \omega_{b} / c=\pi\right.$ in (5)-(6)), we indeed prove the existence of exact travelling breather solutions superposed on an exponentially small periodic tail.

Near the critical parameter value $\tau=\left(V^{\prime \prime}(0)\right)^{-1 / 2}$ (i.e. near the sound velocity), the problem is locally reduced to a finite dimensional reversible system of ordinary differential equations, which admits homoclinic orbits to 0 if $V^{\prime \prime \prime}(0) \neq 0$. These orbits correspond to the FPU solitary waves obtained in reference [27] and satisfy (2) with $p=1$. In addition, the principal part of the reduced system admits homoclinic orbits to quasi-periodic orbits (the persistence of these solutions for the full reduced equation is not yet established). For the FPU system, these solutions should correspond to solitary waves superposed on time-periodic pulsating travelling waves.

In section II A we set the search of travelling breathers into the form of a spatial dynamical system, and give the results on the linearized system. In section II B we treat the general case where the inverse critical velocity $\tau_{k}$ is not $\left(V^{\prime \prime}(0)\right)^{-1 / 2}$ ("sound velocity"). In this section we derive the center manifold reduction, and the study of the solutions of the normal form of the reduced reversible system (we give the main steps of the analysis and shall provide the details in a forthcoming paper [34]). We also give the interpretation of the corresponding solutions of the original system. In section II C we consider the case $\tau \approx\left(V^{\prime \prime}(0)\right)^{-1 / 2}$, which was partly treated in [27], adding the study of cases $p \geq 2$. 


\section{LOCALIZED WAVES IN FERMI-PASTA-ULAM CHAINS}

This section treats the case of the FPU system

$$
\frac{d^{2} u_{n}}{d t^{2}}=V^{\prime}\left(u_{n+1}-u_{n}\right)-V^{\prime}\left(u_{n}-u_{n-1}\right), n \in \mathbb{Z}
$$

with

$$
V(x)=\frac{1}{2} x^{2}+\frac{\alpha}{3} x^{3}+\frac{\beta}{4} x^{4}+O\left(|x|^{5}\right)
$$

(one can fix $V^{\prime \prime}(0)=1$ without loss of generality). We shall analyze small amplitude solutions of (11) satisfying

(2) using the center manifold reduction approach.

The case $p=1$ of (2) is equivalent to fixing $u_{n}(t)=$ $y(x)$ with $x=n-t / \tau$. System (11) is transformed into

$$
\frac{1}{\tau^{2}} \frac{d^{2} y}{d x^{2}}=V^{\prime}(y(x+1)-y(x))-V^{\prime}(y(x)-y(x-1)),
$$

which is a scalar advance-delay or mixed type differential equation. Further references on advance-delay differential equations can be found in [46].

We note that equation (13) can be written in the form $\frac{d I_{1}}{d x}=0$, where

$$
I_{1}=\frac{d y}{d x}-\tau^{2} \int_{0}^{1} V^{\prime}(y(x+v)-y(x+v-1)) d v .
$$

Equation (13) is a particular case in the study which follows, and was studied in [27] for wave velocities $\tau$ close to the sound velocity $\tau=1$, i.e. the maximal velocity of linear phonons.

In the general case of (2) we set $u_{n}(t)=y_{n}(x)$, or equivalently $y_{n}(x)=u_{n}(\tau(n-x))$. This change of variables transforms condition (2) into the periodic boundary condition

$$
y_{n+p}(x)=y_{n}(x)
$$

and system (11) is transformed into the ( $p$-dimensional) system of advance-delay differential equations

$\frac{1}{\tau^{2}} \frac{d^{2} y_{n}}{d x^{2}}=V^{\prime}\left(y_{n+1}(x+1)-y_{n}(x)\right)-V^{\prime}\left(y_{n}(x)-y_{n-1}(x-1)\right)$.

Equation (13) arises as a particular case of (14)-(15) with $p=1$. Note that system (15) admits the following first integral (use condition (14))

$$
I_{p}=\frac{1}{p} \sum_{n=1}^{p} J_{n}
$$

$$
J_{n}=\frac{d y_{n}}{d x}-\tau^{2} \int_{0}^{1} V^{\prime}\left(y_{n+1}(x+v)-y_{n}(x+v-1)\right) d v .
$$

The general case of system (14)-(15) will be treated in section II A. Due to the evenness of (15) in $\tau$ we shall assume $\tau>0$.
Althought the center manifold theorem describes all small amplitude solutions (14)-(15) we shall concentrate on "spatially localized" waves in a generalized sense. These solutions are asymptotic as $x \rightarrow \pm \infty$ to simple shifts $y_{n}=b_{ \pm}\left(b_{ \pm} \in \mathbb{R}\right)$, or to periodic (or quasi-periodic) orbits of small amplitude with respect to central oscillations.

Considering shifted solutions at infinity is necessary because system (15) possesses the invariance $y_{n} \rightarrow y_{n}+b$ $(b \in \mathbb{R})$. The linearized system at $y_{n}=0$ admits a second invariance $y_{n} \rightarrow y_{n}+a x$, which is lost in the nonlinear case. This invariance is replaced by a more subtle one, as discussed in the following remark.

Remark 1. Note that (15) admits the particular solutions $y_{n}(x)=a x, a \in \mathbb{R}$ being an arbitrary constant. These solutions correspond to uniformly compressed or stretched states $u_{n}(t)=a(n-t / \tau)$ (depending whether $a<0$ or $a>0$ ). Moreover, if one chooses $a \in \mathbb{R}$ such that $V^{\prime \prime}(a)>0$ (this holds at least for $a \approx 0$ ), system (14)-(15) has the invariance

$$
\begin{gathered}
y_{n} \rightarrow \tilde{y}_{n}=y_{n}-a x, \quad \tau \rightarrow \sqrt{V^{\prime \prime}(a)} \tau, \\
V \rightarrow V_{a}:=\left(V(x+a)-V^{\prime}(a) x\right) / V^{\prime \prime}(a),
\end{gathered}
$$

where $V_{a}^{\prime}(0)=0, V_{a}^{\prime \prime}(0)=1$. Consequently, from a given class of spatially localized solutions for $\tau \approx \tau_{k}$ one can construct similar solutions of (14)-(15) for $\tau \approx$ $\tau_{k} / \sqrt{V^{\prime \prime}(a)}$, superposed on a uniformly compressed or stretched state. Such solutions have been denoted as "mainly localized" solutions in [27] (case $p=1, \tau \approx 1$ ). They have the form $y_{n}(x)=\tilde{y}_{n}(x, a)+a x$ where $\tilde{y}_{n}$ is a spatially localized solution of (14)-(15) for the modified potential $V_{a}$.

\section{A. Travelling breathers as solutions of a spatial-dynamical system}

\section{Spatial evolution problem}

Instead of treating (14)-(15) directly, we adopt a "dynamical system" point of view by rewriting (15) as an (infinite-dimensional) evolution problem in the spatial coordinate $x$. For this purpose we introduce the new coordinate $v \in[-1,1]$ and functions $Y_{n}(x, v)=y_{n}(x+v)$. We use the notations $\xi_{n}=\frac{d y_{n}}{d x}, \delta_{a} Y_{n}=Y_{n}(x, a)$. The notation $U_{n}(x)(v)=\left(y_{n}(x), \xi_{n}(x), Y_{n}(x, v)\right)^{T}$ indicates our intention to construct $U_{n}$ as a map from $\mathbb{R}$ into some function space living on the $v$-interval $[-1,1]$. System (14)-(15) can then be formulated as an evolution problem in a suitable Banach space. For this purpose we introduce the following Banach spaces $\mathbb{D}$ for $U_{n}(x)$ and $\mathbb{H}$ for $\frac{d U_{n}}{d x}$

$$
\begin{gathered}
\mathbb{H}=\mathbb{R}^{2} \times\left(C^{0}[-1,1]\right), \\
\mathbb{D}=\left\{U=(y, \xi, Y)^{T} \in \mathbb{R}^{2} \times\left(C^{1}[-1,1]\right) / Y(0)=y\right\}
\end{gathered}
$$


with the usual maximum norms. Then we define $Y_{n}(x, v)=y_{n}(x+v), \xi_{n}=\frac{d y_{n}}{d x}$ and $U_{n}=\left(y_{n}, \xi_{n}, Y_{n}\right)^{T}$. The sequence $U(x)=\left(U_{n}(x)\right)_{n \in \mathbb{Z}}$ is a $p$-periodic sequence in $\mathbb{D}$. In the sequel we shall note

$$
U(x) \in \mathbb{D}_{p}=\left\{U \in \mathbb{D}^{\mathbb{Z}} / U_{n+p}=U_{n} \forall n \in \mathbb{Z}\right\} .
$$

Similarly we look for $\frac{d U}{d x}(x)$ in

$$
\mathbb{H}_{p}=\left\{U \in \mathbb{H}^{\mathbb{Z}} / U_{n+p}=U_{n} \forall n \in \mathbb{Z}\right\} .
$$

These spaces are equiped with the usual maximum norms. System (14)-(15) can now be written in the form

$$
\frac{d U}{d x}=L_{\tau} U+\tau^{2} M(U)
$$

where the $n$th term of $L_{\tau} U$ reads

$$
\left(L_{\tau} U\right)_{n}=\left(\begin{array}{c}
\xi_{n} \\
\tau^{2}\left(\delta_{1} Y_{n+1}-2 y_{n}+\delta_{-1} Y_{n-1}\right) \\
\frac{d Y_{n}}{d v}
\end{array}\right)
$$

and the $n$th term of $M(U)$ is given by

$(M(U))_{n}=\left(0, N\left(\delta_{1} Y_{n+1}-y_{n}\right)-N\left(y_{n}-\delta_{-1} Y_{n-1}\right), 0\right)^{T}$, where we have set $N(y)=V^{\prime}(y)-y=O\left(y^{2}\right)$ as $y \rightarrow 0$. It is clear that system (17) is equivalent to the original equation (15) since solutions satisfy $Y_{n}(x, v)=y_{n}(x+v)$. The linear operator $L_{\tau}$ maps $\mathbb{D}_{p}$ into $\mathbb{H}_{p}$ continuously. The nonlinearity $M: \mathbb{D}_{p} \rightarrow \mathbb{D}_{p}$ is analytic in a neighborhood of 0 and $\|M(U)\|_{\mathbb{D}_{p}}=O\left(\|U\|_{\mathbb{D}_{p}}^{2}\right)$.

We note that $L_{\tau}$ and $M$ (hence $L_{\tau}+\tau^{2} M$ ) both commute with the index shift $\sigma$ defined by $(\sigma U)_{n}=U_{n+1}$ (this comes from the invariance $n \rightarrow n+1$ of (15)). Invariant solutions under $\sigma$ correspond in particular to travelling wave solutions of $(11)(U(x) \in \operatorname{Fix}(\sigma)$ is independent of $n$ and its first component $y(x)$ satisfies (13)). If $p$ is even, note that equation (17) is invariant under the symmetry $\sigma^{p / 2}$.

Moreover $L_{\tau}$ and $M$ both anticommute with the reflection $\mathcal{R}$ in $\mathbb{H}_{p}$ given by

$$
(\mathcal{R} U)_{n}=\left(-y_{-n}, \xi_{-n},-Y_{-n}(-v)\right)^{T} .
$$

Therefore, equation (17) is reversible under $\mathcal{R}$. This property is due to the invariance $y_{n} \rightarrow-y_{-n}(-x)$ of (15).

Equation (17) admits the first integral

$$
\mathcal{I}_{\tau}(U)=\frac{1}{p} \sum_{n=1}^{p}\left(\xi_{n}-\tau^{2} \int_{0}^{1} V^{\prime}\left(Y_{n+1}(v)-Y_{n}(v-1)\right) d v\right),
$$

which is issued from the first integral $I_{p}$ of (15). One can check that $\mathcal{I}_{\tau}$ is left invariant by $\sigma$ and $\mathcal{R}$ (use (14)).

Note that particular solutions of (17) are given by $U_{a, b}(x)=(a x+b) \chi_{0}+a \chi_{1}$, where $a, b \in \mathbb{R}$ are arbitrary constants and

$$
\left(\chi_{0}\right)_{n}=(1,0,1)^{T}, \quad\left(\chi_{1}\right)_{n}=(0,1, v)^{T}
$$

(the components of $\chi_{0}, \chi_{1}$ are independent of $n$ ). These solutions originate from the solutions $y_{n}(x)=a x+b$ of (15). Solutions $U_{a, 0}$ are reversible under $\mathcal{R}$ (i.e. $\left.\mathcal{R} U_{a, 0}(-x)=U_{a, 0}(x)\right)$ since $\mathcal{R} \chi_{0}=-\chi_{0}$ and $\mathcal{R} \chi_{1}=\chi_{1}$.

\section{Study of the linearized problem}

We begin by studying the spectrum of $L_{\tau}$, which consists of isolated eigenvalues with finite multiplicities $\left(L_{\tau}\right.$, acting in $\mathbb{H}_{p}$ with domain $\mathbb{D}_{p}$, has a compact resolvent in $\mathbb{H}_{p}$ ). Since $L_{\tau}$ has real coefficients and due to reversibility, its spectrum is invariant under the reflections through the real and the imaginary axis. Due to the periodic boundary condition (14), solving $L_{\tau} U=z U$ (for $z \in \mathbb{C}$ ) is equivalent to searching for solutions of (15) in the form $y_{n}(x)=e^{z x} e^{-2 i \pi m n / p}$ for $m=0, \ldots, p-1$. This yields the dispersion relations

$$
\frac{z^{2}}{\tau^{2}}+2(1-\cosh (z-2 i \pi m / p))=0
$$

As in reference [29], $L_{\tau}$ is not sectorial and the central part of its spectrum (i.e. the set of purely imaginary eigenvalues) is isolated from the hyperbolic part. For purely imaginary eigenvalues $z=i \lambda$, the dispersion relations read

$$
\frac{\lambda^{2}}{\tau^{2}}+2(\cos (\lambda-2 \pi m / p)-1)=0 .
$$

Corresponding linear particle displacements read

$$
u_{n}(t)=a e^{i(q n-\omega t)}+\text { c.c. }
$$

with $q=\lambda-2 \pi m / p, \omega=\frac{\lambda}{\tau}$, hence one recognizes in equation (21) the usual form of the dispersion relation $\omega^{2}=2(1-\cos q)$ of equation (11) linearized at $u_{n}=0$. The case $m=0$ of (21) has been treated in [27]. We note that (21) simplifies into

$$
\frac{|\lambda|}{2 \tau}-\left|\sin \left(\frac{\lambda}{2}-\pi \frac{m}{p}\right)\right|=0 .
$$

Equations (21) and (23) admit the same roots, with identical multiplicities (at most 2) for $\lambda \neq 0$.

Simple roots $\lambda \neq 0$ correspond to simple eigenvalues $i \lambda$ of $L_{\tau}$ for almost all values of $\tau$. They occur in pairs $\pm i \lambda$ corresponding to conjugate modes $m, p-m$. A pair of double semi-simple eigenvalues exists if (23) admits a same root $\lambda$ for $m=m_{1}$ and $m=m_{2}$ with $m_{1} \neq m_{2}$. This occurs for a finite number of parameter values $\tau=$ $\tau_{m_{1} m_{2}}$ defined by

$$
\tau_{m_{1} m_{2}}\left|\cos \left(\frac{\pi}{2 p}\left(m_{1}-m_{2}\right)\right)\right|=\frac{\pi}{2}+\frac{\pi}{2 p}\left(m_{1}+m_{2}\right) .
$$

or

$$
\tau_{m_{1} m_{2}}\left|\sin \left(\frac{\pi}{2 p}\left(m_{1}-m_{2}\right)\right)\right|=\frac{\pi}{2 p}\left(m_{1}+m_{2}\right) .
$$


Double roots $\lambda \neq 0$ correspond to double non semisimple eigenvalues $i \lambda$ of $L_{\tau}$. They occur (in pairs) for critical parameter values $\tau=\tau_{i}(i \geq 1)$, ordered as an unbounded increasing sequence formed by the solutions of

$$
\tau\left|\cos \left(\frac{\lambda}{2}-\pi \frac{m}{p}\right)\right|=1, \quad \frac{\lambda}{2}=\tan \left(\frac{\lambda}{2}-\pi \frac{m}{p}\right),
$$

where $m$ ranges over $0, \ldots, p-1$. Note that $\frac{1}{\tau}$ can be interpreted as a group velocity regarding $(22)\left(\frac{1}{\tau}=\omega^{\prime}(q)\right)$ and the second condition reads $\omega^{\prime}(q)\left(q+2 \pi \frac{m}{p}\right)=\omega(q)$ (phase and group velocities are equal for $m=0$ ).

A closer look at equation (23) indicates that, for $p=1$, $\tau_{1}>\pi$, while for $p \geq 2, \tau_{1}$ is obtained for $m=1, p-1$, $1<\tau_{1}<\pi$ and $\tau_{1} \rightarrow 1$ as $p \rightarrow+\infty$. Moreover, the set of critical velocity values $c_{k}(p)=1 / \tau_{k}(p)(p, k \geq 1)$ densely covers the interval $[0,1]$. In addition, note that (24) implies

$$
\lambda_{k} \sim 2 \tau_{k} \text { as } k \rightarrow+\infty
$$

The following lemma summarizes the evolution of the spectrum as $\tau$ is varied.

Lemma II.1 For $\tau<1$, the spectrum of $L_{\tau}$ on the imaginary axis consists of $p-1$ pairs of simple eigenvalues and the double non semi-simple eigenvalue 0 . Two pairs coincide if $\tau=\tau_{m_{1} m_{2}}$, forming double semi-simple eigenvalues. For $\tau=1$ a pair of real eigenvalues collides at 0 , forming a four-fold eigenvalue. For $1<\tau<\tau_{1}$, the spectrum on the imaginary axis consists of p pairs of simple eigenvalues and the double eigenvalue 0 . For $\tau=\tau_{1}$, two pairs of eigenvalues (originating from the hyperbolic part of the spectrum) collide on the imaginary axis, forming a pair of double non semi-simple eigenvalues $\pm i \lambda$ with $\lambda \neq 0$ (corresponding, for $p \geq 2$, to $m=1, p-1$ ). These pairs split on the imaginary axis for $\tau>\tau_{1}$. Similar eigenvalue collisions occur for all critical values $\tau=\tau_{i}$, increasing (by 4 at each step ) the number of imaginary eigenvalues.

The vectors $\chi_{0}, \chi_{1}$ given in (19) define an eigenvector and a generalized eigenvector associated with the double eigenvalue $0\left(L_{\tau} \chi_{0}=0, L_{\tau} \chi_{1}=\chi_{0}\right)$.

An eigenvector $\zeta$ of $L_{\tau}$ associated with a pair $(\lambda, m)$ $(\lambda \neq 0)$ is given by

$$
(\zeta)_{n}=i e^{-2 i \pi m n / p}\left(1, i \lambda, e^{i \lambda v}\right)^{T} .
$$

With this choice one has $\mathcal{R} \zeta=\bar{\zeta}$. We note that $\sigma \zeta=$ $e^{-2 i \pi m / p} \zeta$ (this explains why eigenvalue collisions related with different modes $m$ correspond to semi-simple eigenvalues, the two colliding modes having different symmetries). If $i \lambda$ is a double eigenvalue, a generalized eigenvector $\eta$ satisfying $\left(L_{\tau}-i \lambda\right) \eta=\zeta$ is given by

$$
(\eta)_{n}=i e^{-2 i \pi m n / p}\left(0,1, v e^{i \lambda v}\right)^{T},
$$

and $\mathcal{R} \eta=-\bar{\eta}$.

\section{B. Cases $\tau \approx \tau_{k}$}

Let us analyze the situation when $\tau \approx \tau_{k}$ and consider $L=L_{\tau_{k}}$. The central part of the spectrum of $L$ consists in

* $N=p+2(k-1)$ pairs of simple eigenvalues $\pm i \lambda_{j}$ $(j=1, \ldots, N), \lambda_{j}$ being associated with $m=m_{j}$ and an eigenvector $\zeta_{j}\left(\left(\zeta_{j}\right)_{n}\right.$ is given by $\left.(26)\right)$,

* 2 pairs of double eigenvalues $\pm i \lambda_{0}, \lambda_{0}$ being associated with $m=m_{0}$, an eigenvector $\zeta_{0}$ given by (26) and a generalized eigenvector $\eta_{0}$ given by $(27)$,

* the double eigenvalue 0 .

In the sequel we denote by $P$ the spectral projection on the $2 N+6$-dimensional central subspace, i.e. the invariant subspace associated with the central part of the spectrum for $\tau=\tau_{k}$. An efficient method for computing $P$ is detailed in [29], [27].

The property of optimal regularity (see [65], hypothesis (ii) p.127) is fulfilled by the affine linearized system

$$
\frac{d U}{d x}=L U+F(x),
$$

where $F(x)$ lies in the range of the nonlinear term $M$ and has the form $(F(x))_{n}=\left(0, g_{n}(x), 0\right)^{T}$. This part of the analysis is similar to [29], lemma 3 p. 448. Alternative methods of proof can be found in references [47], [48].

The property of optimal regularity and the existence of a spectral gap around the imaginary axis allow us to reduce (17) locally to a $2 \mathrm{~N}+6$-dimensional reversible evolution problem on a center manifold [65].

\section{Center manifold reduction}

System (17) is invariant under the shift operator $U \mapsto$ $U+q \chi_{0}$, which corresponds to the invariance $y_{n} \rightarrow$ $y_{n}+q$ in (15). The spectral projection on the generalized eigenspace corresponding to the double eigenvalue $0\left(\tau_{k} \neq 1\right)$ has the form $P_{0} U=\chi_{0}^{*}(U) \chi_{0}+\chi_{1}^{*}(U) \chi_{1}$, where $U_{n}=\left(y_{n}, \xi_{n}, Y_{n}\right)^{T}$,

$$
\chi_{0}^{*}(U)=\frac{1}{p\left(1-\tau_{k}^{2}\right)} \sum_{n=1}^{p}\left(y_{n}-\tau^{2} \int_{-1}^{1} F Y_{n} d v\right),
$$

$F(v)=1-|v|$ and

$\chi_{1}^{*}(U)=\chi_{0}^{*}\left(L_{\tau_{k}} U\right)=\frac{1}{p\left(1-\tau_{k}^{2}\right)} \sum_{n=1}^{p}\left(\xi_{n}+\tau_{k}^{2} \int_{-1}^{1} F^{\prime} Y_{n} d v\right)$

$\left(\chi_{i}^{*}\left(\chi_{j}\right)=\delta_{i j}\right)$. Note that $D \mathcal{I}_{\tau_{k}}(0)=\left(1-\tau_{k}^{2}\right) \chi_{1}^{*}$.

Due to the shift invariance it is natural to decompose any $U \in \mathbb{H}_{p}$ as follows

$$
U=W+q \chi_{0}, \quad \chi_{0}^{*}(W)=0
$$


and we denote by $\tilde{\mathbb{H}}$ the codimension-one subspace of $\mathbb{H}_{p}$ where $W$ lies. We use the similar definition for the subspace $\tilde{\mathbb{D}}$ of $\mathbb{D}_{p}$. Noticing that $\chi_{0}^{*}(M(U))=0$, system (17) becomes

$$
\frac{d W}{d x}=\tilde{L}_{\tau} W+\tau^{2} M(W)
$$

where $\tilde{L}_{\tau} W=L_{\tau} W-\chi_{1}^{*}(W) \chi_{0}$. The operator $\tilde{L}=\tilde{L}_{\tau_{k}}$ acting in $\tilde{\mathbb{H}}$ has the same spectrum as $L_{\tau_{k}}$ except that the eigenvalue 0 is now simple (with eigenvector $\chi_{1}$ ) instead of double. This eigenvalue is linked with the existence of the line of equilibria $W=a \chi_{1}(a \in \mathbb{R})$ of (28), corresponding to the projection of $U_{a, b}$ on $\tilde{\mathbb{D}}$ (one can check that $\left.M\left(a \chi_{1}\right)=0\right)$.

System (28) is supplemented by the scalar equation

$$
\frac{d q}{d x}=\chi_{1}^{*}(W)
$$

Due to the fact that $\mathcal{I}_{\tau}\left(W+q \chi_{0}\right)=\mathcal{I}_{\tau}(W), \mathcal{I}_{\tau}(W)$ also defines a first integral of $(28)$.

Recalling that $P$ is the spectral projection on the $2 N+$ 6-dimensional central space, we shall use the notations $\mathbb{D}_{c}=P \tilde{\mathbb{D}}, \mathbb{D}_{h}=(\mathbb{I}-P) \tilde{\mathbb{D}}, \mathbb{H}_{h}=(\mathbb{I}-P) \tilde{\mathbb{H}}, W_{h}=(\mathbb{I}-P) W$, $W_{c}=P W$. The center manifold reduction theorem [65] applied to system (28) yields the following.

Theorem II.2 Let fix integers $p$ and $k \geq 1$, then for any $m \geq 2$, there exists a neighborhood $\mathcal{U} \times \mathcal{V}$ of $\left(0, \tau_{k}\right)$ in $\tilde{\mathbb{D}} \times \mathbb{R}$ and a map $\psi \in C^{m}\left(\mathbb{D}_{c} \times \mathbb{R}, \mathbb{D}_{h}\right)$ such that the following properties hold for all $\tau \in \mathcal{V}$ (with $\psi(0, \tau)=$ $\left.0, D \psi\left(0, \tau_{k}\right)=0\right)$.

i) If $W: \mathbb{R} \rightarrow \tilde{\mathbb{D}}$ is a solution of (28) and $W(x) \in \mathcal{U}$ for all $x \in \mathbb{R}$, then $W_{h}(x)=\psi\left(W_{c}(x), \tau\right)$ for all $x \in \mathbb{R}$ and $W_{c}: \mathbb{R} \rightarrow \mathbb{D}_{c}$ is a solution of

$$
\frac{d W_{c}}{d x}=\tilde{L} W_{c}+F_{\tau}\left(W_{c}\right)
$$

where $F_{\tau}\left(W_{c}\right)=O\left(\left\|W_{c}\right\|^{2}+\left|\tau-\tau_{k}\right|\left\|W_{c}\right\|\right)$ reads

$$
F_{\tau}\left(W_{c}\right)=P\left[\tilde{L}_{\tau}-\tilde{L}_{\tau_{k}}+\tau^{2} M(.)\right]\left(W_{c}+\psi\left(W_{c}, \tau\right)\right) .
$$

ii) Conversely, if $W_{c}$ is a solution of (30) with $W_{c}(x) \in \mathcal{U}$ for all $x \in \mathbb{R}$, then $W=W_{c}+\psi\left(W_{c}, \tau\right)$ is a solution of (28).

iii) The map $\psi(., \tau)$ commutes with $\mathcal{R}$ and $\sigma$, and (30) is reversible under $\mathcal{R}$ and $\sigma$-equivariant.

Consequently, the $2 N+5$-dimensional reduced equation (30) describes all small amplitude solutions of (28) as $\tau \approx \tau_{k}$. Corresponding solutions of (17) are given by $U=W+q \chi_{0}$ with

$$
\frac{d q}{d x}=\chi_{1}^{*}\left(W_{c}\right)
$$

Note that $\psi\left(a \chi_{1}, \tau\right)=0$ for all $a \approx 0$, due to the fact that $\mathbb{R} \chi_{1}$ is a line of equilibria of (28). In the same way, $\mathbb{R} \chi_{1}$ defines a line of equilibria of (30).

In addition, equation (30) admits the first integral

$$
\begin{aligned}
\mathcal{I}_{c}\left(W_{c}, \tau\right) & =\frac{1}{1-\tau^{2}} \mathcal{I}_{\tau}\left(W_{c}+\psi\left(W_{c}, \tau\right)\right) \\
& =\chi_{1}^{*}\left(W_{c}\right)+O\left(\left\|W_{c}\right\|^{2}+\left|\tau-\tau_{k}\right|\left\|W_{c}\right\|\right),
\end{aligned}
$$

and one can check that $\mathcal{I}_{c}$ is left invariant by $\sigma$ and $\mathcal{R}$.

\section{Normal form}

Now we perform a change of variables close to the identity which simplifies (30) and preserves its symmetries. For that purpose we proceed in two steps.

Firstly we decompose $W_{c}$ into $W_{c}=d \chi_{1}+V_{c}$ (with $\chi_{1}^{*}\left(V_{c}\right)=0$ ) and express the $d$-coordinate with $D=$ $\mathcal{I}_{c}\left(W_{c}, \tau\right)=d+$ h.o.t. Indeed, this equation can be locally inverted in $d=\varphi_{1}\left(D, V_{c}, \tau\right)$, where

$$
\varphi_{1}\left(D, V_{c}, \tau\right)=D+O\left(\left\|\left(D, V_{c}\right)\right\|^{2}+\left|\tau-\tau_{k}\right|\left\|\left(D, V_{c}\right)\right\|\right) .
$$

System (30) takes the form

$$
\frac{d D}{d x}=0, \quad \frac{d V_{c}}{d x}=G\left(V_{c}, D, \tau\right)
$$

where $G(0, D, \tau)=0$.

Secondly we consider the differential equation on $V_{c}$, treating $D, \tau$ as parameters (the eigenvalue 0 is then removed in the $V_{c}$-component). We use a normal form technique (see e.g. [28]), i.e. we perform a polynomial change of variables $V_{c}=\tilde{V}_{c}+\tilde{P}_{\tau, D}\left(\tilde{V}_{c}\right)$ close to the identity which simplifies (32) and preserves its symmetries. In the sequel we set

$$
\tilde{V}_{c}=A \zeta_{0}+B \eta_{0}+\sum_{j=1}^{N} C_{j} \zeta_{j}+c . c .,
$$

where $A, B, C_{j} \in \mathbb{C}$. The normal form computation is similar to [29] (section 6 and appendix 2), to which we refer for details. We compute the normal form at order 3 under the following nonresonance condition

$$
\lambda \cdot r \neq 0 \text { for all } r \in \mathbb{Z}^{N+1} \text { such that } 0<|r| \leq 4,
$$

where $\lambda=\left(\lambda_{0}, \ldots, \lambda_{N}\right)$ and $|r|=\left|r_{0}\right|+\ldots+\left|r_{N}\right|$ (we avoid strong resonances). It is simple to show that (33) is satisfied for $p=1$ and $k=1(N=1)$, and we have checked (33) numerically for $p=2$ and $k=1$. In fact we make the conjecture that (33) is satisfied for any couple $(p, k)$.

The normal form of (32) at order 3 is given in the following lemma.

Lemma II.3 Assume strong resonances do not occur at $\tau=\tau_{k}$, i.e. property (33) is satisfied. The normal form 
of (32) at order 3 reads

$$
\begin{aligned}
\frac{d A}{d x} & =i \lambda_{0} A+B+i A \mathcal{P}\left(|A|^{2}, I, Q, D\right)+\text { h.o.t., } \\
\frac{d B}{d x} & =i \lambda_{0} B+[i B \mathcal{P}+A \mathcal{S}]\left(|A|^{2}, I, Q, D\right)+\text { h.o.t., } \\
\frac{d C_{j}}{d x} & =i \lambda_{j} C_{j}+i C_{j} \mathcal{Q}_{j}\left(|A|^{2}, I, Q, D\right)+\text { h.o.t., } \\
\frac{d D}{d x} & =0
\end{aligned}
$$

$\left(C_{j}\right.$ is considered for $\left.j=1, \ldots N\right)$, where $Q$ denotes the vector $Q=\left(\left|C_{1}\right|^{2}, \ldots,\left|C_{N}\right|^{2}\right), I=i(A \bar{B}-\bar{A} B)$ and $\mathcal{P}, \mathcal{S}, \mathcal{Q}_{j}$ are polynomial functions of their arguments, with real coefficients depending smoothly on $\tau$ for $\tau \approx$ $\tau_{k}$. The principal part of (34) is a cubic polynomial in $A, B, C_{1}, \ldots, C_{N}$, their conjugates, and $D$. Higher order terms are $O\left(\left\|\tilde{V}_{c}\right\|^{4}+\left\|\tilde{V}_{c}\right\||D|\left(\left\|\tilde{V}_{c}\right\|^{2}+D^{2}\right)\right)$. Equation (34) is reversible under the symmetry $\mathcal{R}$ restricted to $\mathbb{D}_{c}$

$$
\mathcal{R}:\left(A, B, C_{1}, \ldots, C_{N}, D\right) \mapsto\left(\bar{A},-\bar{B}, \bar{C}_{1}, \ldots, \bar{C}_{N}, D\right)
$$

and equivariant under the isometry $\sigma$ restricted to $\mathbb{D}_{c}$

$$
\sigma=\operatorname{diag}\left(e^{-2 i \pi \frac{m_{0}}{p}}, e^{-2 i \pi \frac{m_{0}}{p}}, e^{-2 i \pi \frac{m_{1}}{p}}, \ldots, e^{-2 i \pi \frac{m_{N}}{p}}, 1\right) .
$$

The polynomials $\mathcal{P}, \mathcal{S}$ in the normal form (34) have the form

$$
\begin{aligned}
\mathcal{P}= & p_{0}(\tau)+r|A|^{2}+f I \\
& +O\left(|D|+\left|\tau-\tau_{k}\right|\|(A, B)\|^{2}+O\left(\left\|\left(C_{1}, \ldots, C_{N}\right)\right\|^{2}\right),\right. \\
\mathcal{S}= & s_{0}(\tau)+s|A|^{2}+g I \\
& +O\left(|D|+\left|\tau-\tau_{k}\right|\|(A, B)\|^{2}+O\left(\left\|\left(C_{1}, \ldots, C_{N}\right)\right\|^{2}\right),\right.
\end{aligned}
$$

where $r, s, f, g \in \mathbb{R}$ and $p_{0}, s_{0}$ are real-valued functions satisfying $p_{0}\left(\tau_{k}\right)=s_{0}\left(\tau_{k}\right)=0$.

Small amplitude solutions of (34) correspond via theorem II.2 and equation (29) to solutions of the evolution problem (17) having the form

$$
\begin{aligned}
U= & A \zeta_{0}+B \eta_{0}+\sum_{j=1}^{N} C_{j} \zeta_{j}+c . c .+D \chi_{1}+q \chi_{0} \\
& +\tilde{\psi}(A, B, C, \bar{A}, \bar{B}, \bar{C}, D, \tau)
\end{aligned}
$$

where $C=\left(C_{1}, \ldots, C_{N}\right)$ and $\tilde{\psi} \in C^{m}\left(\mathbb{C}^{2 N+4} \times \mathbb{R}^{2}, \tilde{\mathbb{D}}\right)$ satisfies $\tilde{\psi}(0, \tau)=0, D \tilde{\psi}\left(0, \tau_{k}\right)=0$. Note that $\tilde{\psi}$ has a component on $\mathbb{D}_{c}$ due to the normal form transformation (see [28]). We have in addition

$$
\frac{d q}{d x}=D+\chi_{1}^{*}(\tilde{\psi}(A, B, C, \bar{A}, \bar{B}, \bar{C}, D, \tau)) .
$$

The truncated normal form (in which higher order terms are neglected) is integrable $\left(\left|C_{j}\right|^{2}, D\right.$ and $I$ are first integrals), and its study directly follows from the reversible 1:1 resonance case treated in [31]. Small amplitude solutions of the truncated normal form yield approximate (leading order) solutions of (17) (cancel in $\tilde{\psi}$ the terms of order higher than 3 in $\left(A, B, C_{j}, D\right)$ in $(35)$, (36)). For obtaining exact solutions one has to prove the persistence of a given class of solutions of the normal form when higher order terms are taken into account in (34). This problem is specially difficult in the case of homoclinic solutions, as we explain in the next section.

\section{Homoclinic solutions of the normal form system}

For $\tau \approx \tau_{k}$ and $\tau<\tau_{k}, s_{0}(\tau)>0$ and the linearized operator $\tilde{L}_{\tau}$ has four symmetric hyperbolic eigenvalues $\pm \sqrt{s_{0}} \pm i\left(\lambda_{0}+p_{0}\right)$ close to $\pm i \lambda_{0}$. The truncated normal form possesses orbits homoclinic to 0 related to these pairs of eigenvalues if $s<0$. These solutions are given by $C_{j}=D=0$ and

$$
A(x)=r_{0}(x) e^{i\left(\lambda_{0} x+\psi(x)+\theta\right)}, B(x)=\frac{d r_{0}}{d x} e^{i\left(\lambda_{0} x+\psi(x)+\theta\right)},
$$

where $\theta \in \mathbb{R}$ and

$$
\begin{aligned}
r_{0}(x) & =\sqrt{\frac{2 s_{0}}{-s}} \frac{1}{\cosh \left(\sqrt{s_{0}} x\right)}, \\
\psi(x) & =p_{0} x+2 \frac{r}{s} \sqrt{s_{0}} \tanh \left(\sqrt{s_{0}} x\right) .
\end{aligned}
$$

These orbits are reversible under $\mathcal{R}$ if one chooses $\theta$ equal to 0 or $\pi$.

Following the classical normal form computation scheme (see [29] p. 457), we find

$$
s=\frac{\lambda_{0}^{4}}{\left(\tau_{k}^{2}-1\right)^{2}}\left(4 \alpha^{2}\left(1-\frac{1}{2 \tau_{k}^{2}}\right)-3 \beta\left(1-\frac{1}{\tau_{k}^{2}}\right)\right) .
$$

The limit $k \rightarrow+\infty$ in (37) corresponds to travelling breather velocities decaying to 0 , since their inverses $\tau_{k} \rightarrow+\infty$. As $k \rightarrow+\infty, s$ converges towards the finite value $s_{\infty}=16\left(4 \alpha^{2}-3 \beta\right)$ (use $\left.(25)\right)$. The condition $s_{\infty}<0$ is equivalent to the condition $b=3 \beta-4 \alpha^{2}>0$ for the existence of small amplitude static breathers in the FPU chain [32], [33].

We now discuss the sign of $s$ in more detail (we assume that at least one of the coefficients $\alpha, \beta$ is nonzero).

For an even potential $V(\alpha=0), s$ has the sign of $-\beta$ and orbits homoclinic to 0 exist (for the truncated normal form) in the hard potential case $\beta>0$.

The situation is more complex if $\alpha \neq 0$. We note that $s$ has the same sign as $-b+c_{k}^{2}\left(b+2 \alpha^{2}\right)$, where $c_{k}(p)=$ $1 / \tau_{k}(p)$ densely covers the interval $[0,1]$ for $p, k \geq 1$.

On the one hand, if $b<0$ one obtains $s>0$ (since $c_{k}<1$ ) and orbits homoclinic to 0 do not exist for the truncated normal form. This is the case in particular for $\beta \leq 0$ (case of "dark breathers", by analogy with dark solitary waves).

On the other hand, if $b>0$ the condition $s<0$ is satisfied on the velocity interval $0 \leq c_{k}<c_{\max }$, where

$$
c_{\max }^{2}=\frac{b}{b+2 \alpha^{2}}<1 .
$$


We now discuss the question of persistence of reversible solutions homoclinic to 0 , as higher order terms are taken into account in the normal form. For that purpose we restrict the flow of (34) to one on the invariant manifold $D=0$.

In the general case the persistence problem discussed along these lines is still an open question. Relevant tools for this problem are described in references [42], [30].

The particular case $N=1$, in which there is only one pair of purely imaginary eigenvalues $\pm i \lambda_{1}$ in addition to weakly hyperbolic ones, is denoted as $\left(i \lambda_{0}\right)^{2} i \lambda_{1}$ resonance and has been treated in [42]. This case arises only for $p=1$, i.e. for a certain type of travelling wave solutions of (11). Under suitable nonresonance conditions on the eigenvalues, reversible solutions of the normal form, which are homoclinic to periodic orbits, persist above a critical orbit size, which is exponentially small with respect to $\left|\tau-\tau_{k}\right|$. On the contrary, reversible orbits homoclinic to 0 do not persist generically for the full normal form when higher order terms are taken into account.

In what follows we shall examine the general case $N \geq$ 1 intuitively using formal geometric arguments. Since the phase space is now $2 N+4$-dimensional (with $D=$ 0 ), the subspace $\operatorname{Fix}(\mathcal{R})$ is $N+2$-dimensional and the stable manifold $W^{s}(0)$ of the origin (for $\tau<\tau_{k}$ ) is 2dimensional, the intersection of $\operatorname{Fix}(\mathcal{R})$ and $W^{s}(0)$ (at a point $\tilde{V}_{c} \neq 0$ ) should be a phenomenon of codimension $N$. In particular, the lowest codimension value is $p$ and occurs at $\tau \approx \tau_{1}$. Consequently, one can expect that reversible solutions homoclinic to 0 should not persist generically for the full normal form (34).

By analogy with the $\left(i \lambda_{0}\right)^{2} i \lambda_{1}$ resonance case [42], we expect the splitting size between $\operatorname{Fix}(\mathcal{R})$ and $W^{s}(0)$ to be exponentially small as $\tau \rightarrow \tau_{k}$. Exponential smallness can be intuitively understood in the sense that (under suitable nonresonance assumptions) the truncated normal form of (30) at any order admits solutions homoclinic to 0 (the normal form structure is the same as (34)).

Note that one could allow for an additional degree of freedom by searching orbits homoclinic to nonzero equilibria, $D \approx 0$ being treated as a free paramete (this is in connection with Remark 1).

In addition to solutions homoclinic to 0 (for $s<0$, $\tau \approx \tau_{k}$ and $\tau<\tau_{k}$ ), the truncated normal form admits reversible solutions homoclinic to small quasi-periodic orbits, corresponding to $\left|C_{j}\right|=$ const, $j=1, . . N$. Similar solutions exist around nonzero equilibria, provided $D$ is small enough $\left(D=o\left(\left|\tau-\tau_{k}\right|\right)\right.$. By analogy with the $\left(i \lambda_{0}\right)^{2} i \lambda_{1}$ resonance case [42], we conjecture for the full normal form the existence of reversible orbits homoclinic to $N$-dimensional tori, whose sizes could be made exponentially small with respect to $\left|\tau-\tau_{k}\right|$.

Special features arise in the case $p=2$ and $\tau \approx \tau_{1}$ $(N=2)$ if the interaction potential $V$ of $(11)$ is even. Due to the additional invariance $u_{n} \rightarrow-u_{n}$ of (11), equation (17) is also invariant under the symmetry $-\sigma$. Fixed points of $-\sigma$ correspond to solutions of (11) satisfying $u_{n+1}(t)=-u_{n}(t-\tau)$. If one considers the reduced equa- tion (30) on the invariant subspace $\operatorname{Fix}(-\sigma)$, one pair of purely imaginary eigenvalues $\pm i \lambda_{2}$ (corresponding to $m=0$ in (21) and an eigenvector $\zeta_{2}$ invariant under $\sigma$ ) is removed. One has also $\chi_{1}^{*}\left(W_{c}\right)=0$ on $\operatorname{Fix}(-\sigma)$ (since $\chi_{1}$ is invariant under $\sigma$ ), hence the eigenvalue 0 is removed. The invariant subspace $\operatorname{Fix}(-\sigma)$ contains the stable and unstable manifolds of 0 and one recovers the $\left(i \lambda_{0}\right)^{2} i \lambda_{1}$ resonance case (both eigenvalues corresponding to $m=1$ in $(21))$. In addition we note that $\mathcal{I}_{c}=0$ on $\operatorname{Fix}(-\sigma)\left(\mathcal{I}_{c}\right.$ is invariant under $\sigma$ and commutes with $-I$ ).

Consequently, reversible solutions under $\mathcal{R}$ or $-\mathcal{R}$, lying on $\operatorname{Fix}(-\sigma)$ and homoclinic to periodic orbits persist above a critical orbit size, which is exponentially small with respect to $\left|\tau-\tau_{1}\right|$. On the contrary, reversible orbits homoclinic to 0 should not persist generically for the full normal form when higher order terms are taken into account.

This reduction procedure generalises to the case when $p$ is even ( $V$ being symmetric), the relevant symmetry being $-\sigma^{p / 2}$. However, for $\tau \approx \tau_{1}$ there remain $p / 2$ pairs of simple imaginary eigenvalues in addition to the two weakly hyperbolic pairs, and we have no existence result for $p \geq 4$.

\section{Travelling breather solutions}

The solutions of the truncated normal form yield approximate (leading order) solutions of (15)

$$
\begin{aligned}
y_{n}(x) \approx & A(x) i e^{-2 i \pi m_{0} n / p}+\sum_{j=1}^{N}\left(C_{j}(x) i e^{-2 i \pi m_{j} n / p}\right) \\
& + \text { c.c. }+q(x),
\end{aligned}
$$

with $\frac{d q}{d x}=D$ (principal part of equation (36)). Corresponding approximate solutions of the FPU system (11) read $u_{n}(t) \approx y_{n}(n-t / \tau)$.

Leading order solutions of the normal form, which are homoclinic to small quasi-periodic orbits, should constitute the principal part of travelling breather solutions of system (11), superposed at infinity on a quasiperiodic oscillatory tail and a uniformly stretched or compressed state (even for $D=0$, due to the $\tilde{\psi}$ contribution in (36)). We sum up our findings in the following theorem (we exclude the case $p=1$ corresponding to travelling waves).

Theorem II.4 Fix $p \geq 2$ in (2), $k \geq 1$ and consider the near-critical case $\tau \approx \tau_{k}(p)$, and assume $m_{0} \neq 0$. Assume the nonresonance condition (33) holds. Assume $s<0, \tau$ sufficiently close to $\tau_{k}$ with $\tau<\tau_{k}$, and $D \approx 0$ $\left(D=o\left(\left|\tau-\tau_{k}\right|\right)\right.$. The reduced equation (32) written in normal form and truncated at order 4 admits a $N+1-$ parameter family of orbits homoclinic to $N$-dimensional tori with $N=p+2(k-1)$.

Remark 2: Such solutions should correspond to the principal part of travelling breather solutions of system (11), satisfying the commensurability condition (2), and 
superposed at infinity on both a quasiperiodic oscillatory tail and a uniformly stretched or compressed state.

This result is the first step in the proof of the existence of exact travelling breather solutions superposed on an exponentially small oscillatory tail. For obtaining exact solutions of (11) one has to prove the persistence of the above mentioned homoclinic solutions of (34) without h.o.t., when higher order terms are taken into account. This problem is still open in the general case $N \geq 1$ but can be solved in a particular case.

Remark 3: In the case when $m_{0}=0$, we obtain a principal part invariant under $\sigma$, hence a travelling solitary wave superposed on small oscillating pulsating travelling waves.

Theorem II.5 Assume $p=2, s<0$ and $\tau \approx \tau_{1}$ with $\tau<\tau_{1}$. Moreover assume that the potential $V$ is even. Equation (17) is invariant under the symmetry $-\sigma$. The full reduced equation (30) restricted to Fix $(-\sigma)$ admits small amplitude reversible solutions (under $\pm \mathcal{R}$ ) homoclinic to periodic orbits. For a fixed value of $\tau$ (and up to a shift in $x$ ), these solutions occur in a oneparameter family parametrized by the amplitude of periodic orbits. The lower bound of these amplitudes is $O\left(e^{-c /\left|\tau-\tau_{1}\right|^{1 / 2}}\right)(c>0)$. These solutions correspond to exact travelling breather solutions of system (11) superposed at infinity on an oscillatory (periodic) tail and satisfying $u_{n+1}(t)=-u_{n}(t-\tau)$.

Note that the existence of modulated plane waves in FPU chains has been studied by Tsurui [64] using formal multiscale expansions. Under this approximation, the wave envelope satisfies the NLS equation. The condition obtained by the author for the existence of NLS solitons (with group velocity $\omega^{\prime}(q)=1 / \tau_{k}$ ) is exactly the condition $s<0$ derived above.

\section{Case $\tau \approx 1$}

This section is an extension of the study made in [27], where only $p=1$ was considered. Let us follow the same lines as for $\tau \approx \tau_{k}$. In the present case, following Lemma II.1 for $\tau=1$ we have for the linear operator $L_{1}$, in addition to the $p-1$ pairs of simple eigenvalues $\pm i \lambda_{j}$ on the imaginary axis, a quadruple eigenvalue in 0 , with eigenvectors

$$
\begin{aligned}
& \left(\chi_{0}\right)_{n}=(1,0,1)^{T}, \quad\left(\chi_{1}\right)_{n}=(0,1, v)^{T}, \\
& \left(\chi_{2}\right)_{n}=\left(0,0, v^{2} / 2\right)^{T}, \quad\left(\chi_{3}\right)_{n}=\left(0,0, v^{3} / 6\right)^{T},
\end{aligned}
$$

which satisfy

$$
\begin{aligned}
L_{1} \chi_{0} & =0, \quad L_{1} \chi_{j}=\chi_{j-1}, \quad j=1,2,3 \\
\mathcal{R} \chi_{0} & =-\chi_{0}, \mathcal{R} \chi_{1}=\chi_{1}, \mathcal{R} \chi_{2}=-\chi_{2}, \mathcal{R} \chi_{3}=\chi_{3} .
\end{aligned}
$$

The spectral projection on the 4-dimensional generalized eigenspace belonging to 0 has the form

$$
P_{0} U=\sum_{0 \leq j \leq 3} \chi_{j}^{*}(U) \chi_{j},
$$

where

$$
\begin{gathered}
U_{n}=\left(y_{n}, \xi_{n}, Y_{n}\right)^{T}, \chi_{j}^{*}\left(\chi_{i}\right)=\delta_{i j}, \\
\chi_{0}^{*}(U)=\frac{2}{5 p} \sum_{1 \leq n \leq p}\left(y_{n}-\int_{-1}^{1} G Y_{n} d v\right), \\
G(v)=1-|v|-5(1-|v|)^{3}, \\
\chi_{1}^{*}(U)=\chi_{0}^{*}\left(L_{1} U\right)=\frac{2}{5 p} \sum_{1 \leq n \leq p}\left(\xi_{n}+\int_{-1}^{1} G^{\prime} Y_{n} d v\right), \\
\chi_{2}^{*}(U)=\chi_{1}^{*}\left(L_{1} U\right)=\frac{-12}{p} \sum_{1 \leq n \leq p}\left(y_{n}-\int_{-1}^{1} F Y_{n} d v\right), \\
\chi_{3}^{*}(U)=\chi_{2}^{*}\left(L_{1} U\right)=\frac{-12}{p} \sum_{1 \leq n \leq p}\left(\xi_{n}+\int_{-1}^{1} F^{\prime} Y_{n} d v\right),
\end{gathered}
$$

where we note that for $v \neq 0, G^{\prime \prime}=-30 F=-30(1-|v|)$, and $\chi_{3}^{*}\left(L_{1} U\right)=0$.

\section{Center manifold reduction}

In the same way as in section II B we use the decomposition of any $U \in \mathbb{H}_{p}$ as

$$
U=W+q \chi_{0}, \quad \chi_{0}^{*}(W)=0,
$$

and we obtain a system (28), (29), where the operator $\widetilde{L}$ acting in $\widetilde{\mathbb{H}}$ has the same spectrum as $L_{1}$ except that the eigenvalue 0 is triple (eigenvector $\chi_{1}$ ). Theorem II.2 still applies in this case, replacing $\tau_{k}$ by $1, W_{c}$ lies in a $2(\mathrm{p}-1)+3$ - dimensional space, and the first integral (18) becomes

$$
\begin{aligned}
\mathcal{I}_{c}\left(W_{c}, \tau\right) & =-\frac{1}{12} \mathcal{I}_{\tau}\left(W_{c}+\psi\left(W_{c}, \tau\right)\right) \\
& =\chi_{3}^{*}\left(W_{c}\right)+O\left(\left\|W_{c}\right\|^{2}+(|\tau-1|) \| W_{c}||\right) .
\end{aligned}
$$

\section{Normal form}

Following the same structure as in section II B, we decompose $W_{c}$ as follows

$$
W_{c}=d \chi_{3}+V_{c}, \quad \chi_{3}^{*}\left(V_{c}\right)=0
$$

and use coordinate $D$ instead of $d$, where, as above

$$
D=\mathcal{I}_{c}\left(W_{c}, \tau\right), \quad d=\varphi_{3}\left(D, V_{c}, \tau\right)
$$

$$
\varphi_{3}\left(D, V_{c}, \tau\right)=D+O\left(\left\|\left(D, V_{c}\right)\right\|^{2}+|\tau-1|\left\|\left(D, V_{c}\right)\right\|\right) .
$$

Then system (32) is still valid, except that

$$
G(0, D, \tau) \neq 0
$$


since the line of solutions for (28) is $\mathbb{R} \chi_{1}$ and not $\mathbb{R} \chi_{3}$. As above, $D$ and $\tau$ are treated as parameters, and 0 is now a double eigenvalue of the linearized system in $V_{c}$, for $\tau=1, D=0$. Then we have the following (see [27], [28] where the invariant $D$ corresponds there to the first integral $H$ of the normal form)

Lemma II.6 Assume strong resonances do not occur at $\tau=1$, i.e. property (33) is satisfied for $\lambda_{j}, j=1, \ldots p-1$. Denoting by $A, B$ the components of $\widetilde{V}_{c}$ (after the polynomial change of variables) along $\chi_{1}$ and $\chi_{2}$, the normal form of (32) at order 3 reads

$$
\begin{aligned}
\frac{d A}{d x} & =B \\
\frac{d B}{d x} & =D+A \phi(A, Q, D, \tau)+\text { h.o.t. } \\
\frac{d C_{j}}{d x} & =i \lambda_{j} C_{j}+i C_{j} \mathcal{Q}_{j}(A, Q, D, \tau)+\text { h.o.t. } \\
\frac{d D}{d x} & =0,
\end{aligned}
$$

$\left(C_{j}\right.$ is considered for $\left.j=1, \ldots p-1\right)$, where $Q$ denotes the vector $Q=\left(\left|C_{1}\right|^{2}, \ldots,\left|C_{N}\right|^{2}\right)$, and $\phi$ and $\mathcal{Q}_{j}$ are polynomial functions of their arguments $A, B, C_{j}, D$, with real coefficients depending smoothly on $\tau$ for $\tau \approx 1$. The principal part of (39) is a cubic polynomial in $C_{1}, \ldots, C_{N}$, their conjugates, and $A, B, D$. Higher order terms are $O\left(\left\|\tilde{V}_{c}\right\|^{4}+\left\|\tilde{V}_{c}\right\||D|\left(\left\|\tilde{V}_{c}\right\|^{2}+D^{2}\right)\right)$. Equation (39) is reversible under the symmetry $\mathcal{R}$ restricted to $\mathbb{D}_{c}$

$$
\mathcal{R}:\left(A, B, C_{1}, \ldots, C_{N}, D\right) \mapsto\left(A,-B, \bar{C}_{1}, \ldots, \bar{C}_{N}, D\right),
$$

and equivariant under the isometry $\sigma$ restricted to $\mathbb{D}_{c}$

$$
\sigma=\operatorname{diag}\left(1,1, e^{-2 i \pi \frac{m_{1}}{p}}, \ldots, e^{-2 i \pi \frac{m_{N}}{p}}, 1\right), N=p-1 .
$$

The polynomial $\phi$ has the form

$$
\begin{aligned}
\phi(A, Q, D, \tau)= & \nu+a A+ \\
& +b A^{2}+\sum_{1 \leq j \leq p-1} b_{j}\left|C_{j}\right|^{2}
\end{aligned}
$$

where $\nu, a, b, b_{j}$ are smooth functions of $(D, \tau)$ near $(0,1)$, and $\nu(D, 1)=0$,

$$
\begin{aligned}
& \nu(D, \tau)=24(1-\tau)\{1+O(|D|+|1-\tau|)\}, \\
& a(D, \tau)=-12 \alpha\{1+O(|1-\tau|)\}+O(|D|), \\
& b(D, \tau)=-12 \beta\{1+O(|1-\tau|)\}+O(|D|), \text { if } \alpha=0 .
\end{aligned}
$$

(see [27] for the computation of the coefficients $a$ and $b$ ). Small amplitude solutions of (39) correspond, via theorem II.2 and equation (29) to solutions of the evolution problem (17) having the form

$$
\begin{aligned}
U= & A \chi_{1}+B \chi_{2}+\sum_{j=1}^{N} C_{j} \zeta_{j}+c . c .+D \chi_{3}+q \chi_{0} \\
& +\tilde{\psi}(A, B, C, \bar{C}, D, \tau)
\end{aligned}
$$

where $C=\left(C_{1}, \ldots, C_{p-1}\right)$ and $\tilde{\psi} \in C^{m}\left(\mathbb{R}^{2} \times \mathbb{C}^{2 p-2} \times\right.$ $\left.\mathbb{R}^{2}, \tilde{\mathbb{D}}\right)$ satisfies $\tilde{\psi}(0, \tau)=0, D \tilde{\psi}(0,1)=0$. Note that $\tilde{\psi}$ has a component on $\mathbb{D}_{c}$ due to the normal form transformation (see [28]) and that the line of steady solutions of (28) corresponds to

$$
B=0, D+A \phi(A, 0, D, \tau)+\text { h.o.t. }=0
$$

which gives a component on $\chi_{3}$ anihilated by $\tilde{\psi}(A, 0,0,0, D, \tau)$. We have in addition

$$
\frac{d q}{d x}=A+\chi_{1}^{*}(\tilde{\psi}(A, B, C, \bar{C}, D, \tau)) .
$$

The truncated normal form (in which higher order terms are neglected) is integrable $\left(\left|C_{j}\right|^{2}\right.$ and $D$ are first integrals), and its study directly follows from the reversible $0^{2+}$ singularity case (see for example [28]). Small amplitude solutions of the truncated normal form yield approximate (leading order) solutions of (17) (cancel in $\tilde{\psi}$ the terms of order higher than 3 in $\left(A, B, C_{j}, D\right)$ in (40), (41)). For obtaining exact solutions one has to prove the persistence of a given class of solutions of the normal form when higher order terms are taken into account in (39).

\section{Homoclinic solutions of the normal form system}

For $\tau \approx 1$ and $\tau<1$, then $\nu>0$ and the linearized operator $\widetilde{L}_{\tau}$ has one simple eigenvalue 0 , and a pair of real symmetric eigenvalues $\pm \sqrt{\nu}$ near 0 . For $4 a D<\nu^{2}$, the truncated normal form (39) possesses two equilibria ( $\left.A=A_{0}^{ \pm}, B=0, C_{j}=0\right)$, one hyperbolic, one elliptic, and an orbit homoclinic to the hyperbolic equilibrium. For $D=0$, and $\alpha \neq 0$ this orbit is homoclinic to 0 and given by

$$
\begin{aligned}
& A(x)=-\frac{3 \nu(0, \tau)}{2 a(0, \tau) \cosh ^{2}\left(\nu^{1 / 2} x / 2\right)} \\
& B(x)=A^{\prime}(x), \quad C_{j}=0, j=1, \ldots p-1 .
\end{aligned}
$$

For $\alpha=0, \tau<1$ and $b<0$, and $D=0$, orbits homoclinic to 0 are given by

$$
\begin{aligned}
& A(x)= \pm \frac{(-2 \nu / b)^{1 / 2}}{\cosh \left(\nu^{1 / 2} x\right)} \\
& B(x)=A^{\prime}(x), \quad C_{j}=0, j=1, \ldots p-1,
\end{aligned}
$$

while for $\alpha=0, \tau>1$ and $b>0$ we have for $D=0$ a pair of symmetric front solutions, given by

$$
\begin{aligned}
& A(x)= \pm(-\nu / b)^{1 / 2} \tanh \left((-\nu / 2)^{1 / 2} x\right), \\
& B(x)=A^{\prime}(x), \quad C_{j}=0, j=1, \ldots p-1,
\end{aligned}
$$

while for $D \neq 0$ we have orbits homoclinic to one of the two hyperbolic fixed points (see [27] for the details with respect to the dependency in function of $D$ ). 
It is shown in [27] that all the homoclinics and front solutions of the normal form persist when one considers the complete system, when $p=1$, i.e. when we only look for travelling waves. This also corresponds to the persistence of such solutions for larger $p$, in considering solutions in the subspace invariant under the mapping $\sigma$.

Now, we see that the cubic normal form also admits a family of orbits homoclinic to quasi-periodic solutions, with $\left|C_{j}\right|=$ const where these constants are small enough (change $\nu$ in the above formulas into $\nu+\sum b_{j}\left|C_{j}\right|^{2}$ ). As for the case $\tau \approx \tau_{k}$, we are not able to prove the persistence of such homoclinics, except for the case $p=2$ where there is only one coordinate $C_{1} \in \mathbb{C}$ : in such a case we recover the study made in [42] of the reversible $0^{2+} i \lambda_{1}$ singularity of a reversible vector field, where there exists a family of two reversible orbits homoclinic to periodic orbits, provided that their size is not smaller than a quantity which is exponentially small with respect to $|1-\tau|$. Note that in this latter case, the action of the map $\sigma$ exchanges the two orbits in changing $C_{1}$ into $-C_{1}$, and that the orbit homoclinic to 0 persists, due to its invariance under $\sigma$.

\section{Solitary waves superposed to small oscillatory pulsating} travelling waves

The solutions of the cubic normal form yield approximate solutions with leading order

$$
\begin{aligned}
y_{n}(x) & =\sum_{1 \leq j \leq p-1} C_{j}(x) i e^{-2 i \pi m_{j} n / p}+\text { c.c. }+q(x) \\
\frac{d q}{d x} & =A(x)
\end{aligned}
$$

and corresponding solutions of the FPU system (11) read $u_{n}(t) \approx y_{n}(n-t / \tau)$.

Leading order solutions of the normal form, which are homoclinic to small quasi-periodic orbits, should consist in a principal part of solitary waves, solutions of system (11), superposed to a quasiperiodic oscillatory pulsating travelling wave, and to a uniformly stretched or compressed state (see (41)). We sum up our findings in the following theorem (we exclude the case $p=1$ corresponding to travelling waves).

Theorem II.7 Fix $p \geq 2$ in (2), and consider the nearcritical case $\tau \approx 1$. Assume the nonresonance condition (33) holds. Assume $\tau$ sufficiently close to 1 , and $D \approx 0$ $(D=o(|\tau-1|)$. In the general case, the reduced equation (32) written in normal form and truncated at order 4 admits $p$ - parameter family of orbits homoclinic to $p$-1-dimensional tori. In the case of an even and hardening potential, we have for $\tau<1$ a p-parameter family of symmetric homoclinics to $(p-1)-$ dimensional tori, while in case of an even softening potential, we have for $\tau>1$ a $(p-1)$ - parameter family of symmetric fronts connecting symmetric $(p-1)$ - dimensional tori, in addition to a $p$ - parameter family of orbits homoclinic to $(p-1)-$ dimensional tori. For $p=2$, the above result holds in replacing the tori by periodic orbits, and holds true for the full system. The lower bound of the amplitudes of limiting periodic orbits is $O\left(e^{-c /|\tau-1|}\right),(c>0)$. These last solutions are superpositions of a solitary wave or a front, solution of system (11), with small oscillatory pulsating travelling waves.

Remark 2 above is still valid for $p \geq 3$, i.e. such solutions should correspond to a pure travelling principal part solution of system (11), superposed on both a small quasiperiodic oscillatory pulsating travelling wave and a uniformly stretched or compressed state.

Acknowledgements. G.J. is grateful to Serge Aubry for his hospitality at the Laboratoire Léon Brillouin (CEA Saclay, France) and stimulating discussions.
[1] M.J. Ablowitz and J.F. Ladik. Nonlinear differentialdifference equations and Fourier analysis, J. Math. Phys. 17, 1011-1018 (1976).

[2] M.J. Ablowitz, Z. Musslimani and G. Biondini. Methods for discrete solitons in nonlinear lattices, Phys. Rev. E 65 (2002).

[3] M.J. Ablowitz and Z. Musslimani. Discrete spatial solitons in a diffraction-managed nonlinear waveguide array : a unified approach, Physica D 184, 276-303 (2003).

[4] A.A. Aigner, A.R. Champneys and V.M. Rothos. A new barrier to the existence of moving kinks in FrenkelKontorova lattices, Physica D 186, 148-170 (2003).

[5] S. Aubry, G. Kopidakis and V. Kadelburg. Variational proof for hard discrete breathers in some classes of hamiltonian dynamical systems, Discrete and Continuous Dynamical Systems B 1, 271-298 (2001).

[6] S. Aubry, T. Crétégny. Mobility and reactivity of discrete breathers, Physica D 119, 34-46 (1998).

[7] A. Berger, R.S. MacKay, V.M. Rothos. A criterion for non-persistence of travelling breathers for perturbations of the Ablowitz-Ladik lattice. To appear in Discrete Cont. Dyn. Sys. B.

[8] S.R. Bickham, S.A. Kiselev and A.J. Sievers. Stationary and moving intrinsic localized modes in one-dimensional monoatomic lattices with cubic and quartic anharmonicity, Phys. Rev. B 47, 21 (1993).

[9] T. Dauxois, M. Peyrard and A.R. Bishop. Dynamics and thermodynamics of a nonlinear model for DNA denaturation, Phys Rev E 47, n. 1, 684 (1993).

[10] T. Dauxois, M. Peyrard and C.R. Willis. Discreteness effects on the formation and propagation of breathers in nonlinear Klein-Gordon equations, Phys. Rev. E 48, 4768 (1993).

[11] D.B. Duncan, J.C. Eilbeck, H. Feddersen and J.A.D. 
Wattis. Solitons in lattices, Physica D 68, 1-11 (1993).

[12] J.C. Eilbeck and R. Flesch. Calculation of families of solitary waves on discrete lattices, Physics Letters A 149, 200-202 (1990).

[13] E. Fermi, J. Pasta and S. Ulam, Studies of nonlinear problems, Technical Report LA-1940, Los Alamos National Laboratory, 1955.

[14] S. Flach and K. Kladko. Moving discrete breathers ?, Physica D 127, 61-72 (1999).

[15] S. Flach and C.R. Willis. Movability of localized excitations in Nonlinear Discrete systems: a separatrix problem, Phys. Rev. Lett. 72, 1777 (1994).

[16] S. Flach, Y. Zolotaryuk and K. Kladko. Moving kinks and pulses: an inverse method, Phys. Rev. E 59, 6105-6115 (1999).

[17] S. Flach and C.R. Willis. Discrete Breathers, Physics Reports 295, 181-264 (1998).

[18] N. Flytzanis, St. Pnevmatikos and M. Remoissenet. Kink, breather, and asymmetric envelope or dark solitons in nonlinear chains. I monoatomic chain, J. Phys. $C$ Solid State Phys. 18, 4603-4629 (1985).

[19] G. Friesecke and K. Matthies. Atomic-scale localization of high-energy solitary waves on lattices. Physica D 171, 211-220 (2002).

[20] G. Friesecke, R.L. Pego, Solitary waves on FPU lattices : I. Qualitative properties, renormalization and continuum limit, Nonlinearity 12, 1601-1627 (1999).

[21] G. Friesecke, R.L. Pego, Solitary waves on FPU lattices : II. Linear implies nonlinear stability, Nonlinearity 15, 1343-1359 (2002).

[22] G. Friesecke, R.L. Pego, Solitary waves on FPU lattices : III. Howland-type Floquet theory, Nonlinearity 17, 207227 (2004).

[23] G. Friesecke, R.L. Pego, Solitary waves on FPU lattices : IV. Proof of stability at low energy, Nonlinearity 17, 229-251 (2004).

[24] G. Friesecke, J.A Wattis. Existence theorem for solitary waves on lattices, Commun. Math. Phys. 161, 391-418 (1994).

[25] J. Giannoulis, A. Mielke. The nonlinear Schrödinger equation as a macroscopic limit for an oscillator chain with cubic nonlinearities, Nonlinearity 17, p. 551-565 (2004).

[26] J. Giannoulis, A. Mielke. Macroscopic dynamics of an oscillator chain described by the nonlinear Schrödinger equation, preprint, Institut für Analysis, Dynamik und Modellierung, University of Stuttgart (2004).

[27] G. Iooss. Travelling waves in the Fermi-Pasta-Ulam lattice, Nonlinearity 13 849-866 (2000).

[28] G. Iooss, M. Adelmeyer. Topics in bifurcation theory and applications, Adv. Ser. Nonlinear Dynamics 3, World Sci. (1992).

[29] G. Iooss, K. Kirchgässner. Travelling waves in a chain of coupled nonlinear oscillators, Commun. Math. Phys. 211, 439-464 (2000).

[30] G. Iooss, E. Lombardi. Polynomial normal forms with exponentially small remainder for analytic vector fields. Preprint Institut Non Linéaire de Nice, 2004.

[31] G. Iooss, M-C Pérouème. Perturbed homoclinic solutions in reversible 1:1 resonance vertor fields, J. Diff. Eqs. 102, 62-88 (1993).

[32] G. James. Existence of breathers on FPU lattices. C.R.Acad.Sci.Paris, t. 332, Série I (2001), p. 581-586.

[33] G. James. Centre manifold reduction for quasilinear dis- crete systems, J. Nonlinear Sci., vol. 13, 1 , p. 27-63 (2003).

[34] G. James and G. Iooss, in preparation (2004).

[35] G. James and P. Noble. Breathers on diatomic FermiPasta-Ulam lattices, Physica D 196, p. 124-171 (2004).

[36] G. James and Y. Sire. Travelling breathers with exponentially small tails in a chain of nonlinear oscillators. To appear in Commun. Math. Phys. (2004).

[37] L.A. Kalyakin. Long wave asymptotics. Integrable equations as asymptotic limits of non-linear systems. Russian Math. Surveys 44 (1), 3-42 (1989).

[38] M. Kastner and J-A. Sepulchre. Effective Hamiltonian for traveling discrete breathers in the FPU chain. Submitted to Discrete Cont. Dyn. Sys. B (2003).

[39] K. Kirchgässner. Wave solutions of reversible systems and applications, Journal of Differential Equations 45, 113-127 (1982).

[40] T.A. Kontorova and Ya.J. Frenkel, On the theory of plastic deformation and twinning, Zhurn. Ehksper. Teor. Fiz. 8 (1938), p. $1340-1348$.

[41] R. Livi, M. Spicci and R.S. Mackay. Breathers on a diatomic FPU chain, Nonlinearity 10, p. 1421-1434 (1997).

[42] E. Lombardi. Oscillatory integrals and phenomena beyond all algebraic orders with applications to homoclinic orbits in reversible systems, Lecture Notes in Mathematics, Springer-Verlag, vol. 1741 (2000).

[43] E. Lombardi. Phenomena beyond all orders and bifurcations of reversible homoclinic connections near higher resonances, Peyresq Lectures on Nonlinear Phenomena, Kaiser R. and Montaldi J. (eds.), World Scientific, p. 161-200 (2000).

[44] R.S. Mackay, S. Aubry. Proof of existence of breathers for time-reversible or Hamiltonian networks of weakly coupled oscillators, Nonlinearity 7 1623-1643 (1994).

[45] R.S. MacKay, J-A. Sepulchre. Effective Hamiltonian for travelling discrete breathers, J. Phys. A 35, 3985-4002 (2002).

[46] J. Mallet-Paret. The global structure of traveling waves in spatially discrete systems, J. Dyn. Diff. Eqs. 11, n.1 (1999).

[47] J. Mallet-Paret. The Fredholm alternative for functional differential equations of mixed type, J. Dyn. Diff. Eqs. 11, n.1 (1999).

[48] J. Mallet-Paret and S. Verduyn Lunel. Exponential dichotomies and Wiener-Hopf factorizations for mixed type functional differential equations (2001).

[49] A. Mielke. Reduction of quasilinear elliptic equations in cylindrical domains with applications, Math. Meth. Appl. Aci. 10, 51-66 (1988).

[50] A. Mielke. Über maximale $L^{p}$-Regularität für Differentialgleichungen in Banach und Hilbert Raümen, Math. Ann. 277, 121-133 (1987).

[51] M. Remoissenet. Low-amplitude breather and envelope solitons in quasi-one-dimensional physical models, Phys. Rev. B 33, number 4, 2386 (1986).

[52] K.W. Sandusky, J.B. Page and K.E. Schmidt. Stability and motion of intrinsic localized modes in nonlinear periodic lattices, Phys. Rev. B 46, 10 (1992).

[53] A.V. Savin, Y. Zolotaryuk, J.C. Eilbeck. Moving kinks and nanopterons in the nonlinear Klein-Gordon lattice, Physica D 138, 267-281 (2000).

[54] J-A. Sepulchre. Energy barriers in coupled oscillators: from discrete kinks to discrete breathers, Proceedings 
of the Conference on Localization and Energy Transfer in Nonlinear Systems, June 17-21, 2002, San Lorenzo de El Escorial, Madrid, Spain; World Scientific, Eds L. Vazquez, R.S. MacKay, M-P. Zorzano, 102-129 (2003).

[55] A.J. Sievers and S. Takeno, Intrinsic localized modes in anharmonic crystals, Phys. Rev. Lett. 61 (1988), p.970973.

[56] G. Schneider and C.E. Wayne. Counter-propagating waves on fluid surfaces and the continuum limit of the Fermi-Pasta-Ulam model. In B. Fiedler, K. Gröger and J. Sprekels, editors, International Conference on Differential Equations Appl., 5 (1), 69-82 (1998).

[57] Y. Sire, G. James. Travelling breathers in Klein-Gordon chains, C. R. Acad. Sci. Paris, Ser. I 338, 661-666 (2004).

[58] Y. Sire, G. James. Computation of large amplitude travelling breathers in Klein-Gordon chains, in preparation (2004).

[59] Y. Sire. Travelling breathers in Klein-Gordon lattices as homoclinic orbits to $p$-tori. In preparation.
[60] D. Smets, M. Willem. Solitary waves with prescribed speed on infinite lattices, J. Funct. Anal. 149, 266-275 (1997).

[61] J. Szeftel, G. Huang and V. Konotop. On the existence of moving breathers in one-dimensional anharmonic lattices, Physica D 181, 215-221 (2003).

[62] S. Takeno and K. Hori. A propagating self-localized mode in a one-dimensional lattice with quartic anharmonicity, J. Phys. Soc. Japan 59, 3037 (1990).

[63] A.J. Sievers and S. Takeno. Intrinsic localized modes in anharmonic crystals, Phys. Rev. Lett. 61, 970-973 (1988).

[64] A. Tsurui. Wave modulations in anharmonic lattices, Progress of Theoritical Physics, 48 , number 4, 1196-1203 (1972).

[65] A. Vanderbauwhede, G. Iooss. Center manifold theory in infinite dimensions. Dynamics reported 1, new series,125163 (1992). 